\title{
Minimum Convex Partition of Polygonal Domains by Guillotine Cuts*
}

\author{
H. Martini ${ }^{1}$ and V. Soltan ${ }^{2}$ \\ ${ }^{1}$ Fakultät für Mathematik, TU Chemnitz-Zwickau, \\ D-09009 Chemnitz, Germany \\ ${ }^{2}$ Mathematical Institute of the Academy of Sciences of Moldova, \\ MD-2028 Chişinău, Moldova
}

\begin{abstract}
Let $P$ be a multiply connected polygonal domain with holes (possibly degenerate) and let $\mathcal{F}$ be a certain family of directions in the plane. We give a formula for the minimum number of convex pieces in which $P$ is partitioned with the help of guillotine cuts along the directions of $\mathcal{F}$.
\end{abstract}

\section{Introduction}

The problems of partitioning a polygon (with its interior) into a minimum number of convex pieces are well studied in computational geometry (see, for instance, [3], [10], [12], and [14]). Formulas presenting some estimates for the minimum number of convex pieces are given for partitioning triangles (see [4]), trapezoids (see [1] and [19]), rectangles (see [6], [11], and [18]), and convex polygons (see [2]). For the case of minimum convex partitions obtained by cuts along a given family of directions, the respective formulas are given in [15] and [17] in terms of $d$-convexity.

Any convex partition of a polygon $P$ can be obtained by drawing within $P$ finitely many closed line segments. By varying the conditions on their placement, we can consider different types of partitions. For example, if we draw only diagonals of $P$, we obtain socalled partitions without Steiner points (see, e.g., [10] and [12]). If we give preference to segments along certain fixed directions, we obtain partitions into rectangles, trapezoids, etc. In what follows we consider the last approach. Observe that if the prescribed family of directions consists of all directions in the plane, then all possible convex partitions are taken into consideration.

\footnotetext{
* The second author was supported by Deutsche Forschungsgemeinschaft.
} 
More precisely, our purpose is to study the following problem. For a given multiply connected, polygonal domain $P$ with holes (possibly degenerate) and a certain family $\mathcal{F}$ of directions in the plane, determine the minimum number of convex pieces into which $P$ can be partitioned with the help of guillotine cuts (i.e., of linear cuts from boundary points to boundary points, see Definitions 6 and 7 for an exact description) along directions from $\mathcal{F}$.

The paper is organized as follows. In Section 2 we give a description of polygonal domains in order to clarify the notion of a multiply connected polygon with (degenerate) holes. Next (in Section 3) we introduce the notion of local nonconvexity of a polygonal domain, and in Section 4 we define convex guillotine partitions of such domains. Furthermore (see Section 5), we deal with a "naive" guillotine partition into convex pieces, which we use in Section 7 as the procedure for minimum convex guillotine partition. In Section 6 we introduce the notions of a compatible family of diagonals of a polygonal domain as well as the effective number of such a family; both of which are important for minimum convex partition.

\section{Description of Polygonal Domains}

In a standard way, a simple polygon is a compact set in the plane $E$ bounded by a polygonal contour, i.e., by a closed, non-self-intersecting curve consisting of finitely many closed line segments. A polygon with holes is a connected, compact set in the plane whose boundary consists of an exterior polygonal contour and of finitely many interior polygonal contours, which are pairwise disjoint, noninclusive, and determine holes.

We extend the notion of a polygon in order to consider also multiply connected domains with degenerate holes. Thus, by a polygon $P$ we mean a compact set (maybe, multiply connected) with the nonempty interior int $P$ being dense in $P$, and the boundary bd $P$ consisting of finitely many closed line segments. In this way the boundary bd $P$ of $P$ consists of finitely many simple polygonal contours satisfying the following conditions: (1) any two of them are not interlaced, i.e., they may be situated either one inside the other or mutually noninclusive; (2) they may have common vertices but no common line segment.

Clearly, any polygon $P$ can be represented as a list of contours satisfying the above conditions. In this way, the boundary bd $P$ of $P$ is exactly the point-set union of the contours, and the interior int $P$ of $P$ is the set of points each lying inside an odd number of contours.

By a hole of a polygon $P$ we mean any bounded component of the complement $E \backslash P$, and the exterior of $P$ is the unbounded component of $E \backslash P$. Clearly, any hole and the exterior of $P$ are open sets.

As a next step, we introduce the notion polygonal domain with degenerate holes. To do this, we need some preliminary notions.

Assume that some points $v_{1}, v_{2}, \ldots, v_{k}$ and some closed line segments $s_{1}, s_{2}, \ldots, s_{t}$ are placed inside a polygon $P$ such that the following conditions hold:

(1) All the points $v_{1}, v_{2}, \ldots, v_{k}$ belong to int $P \backslash\left(s_{1} \cup s_{2} \cup \cdots \cup s_{t}\right)$.

(2) The relative interior of every line segment $s_{i}$ lies in int $P$. 
(3) If some line segments $s_{i}, s_{j}$ have a common point, then it is a vertex for both $s_{i}, s_{j}$.

The point-set union of these points and segments is called the ornament of $P$ and is denoted by Or $P$ :

$$
\text { Or } P:=\left(\bigcup_{i=1}^{k} v_{i}\right) \cup\left(\bigcup_{i=1}^{t} s_{i}\right) .
$$

A polygon $P$, considered together with a certain ornament Or $P$, is called a polygonal domain.

Definition 1. For a given polygonal domain $P$, the sets

$$
\operatorname{Bd} P:=\text { Or } P \cup \text { bd } P, \quad \text { Int } P:=P \backslash \operatorname{Bd} P
$$

are called the formal boundary and the formal interior of $P$, respectively. A bounded component of the complement $E \backslash \operatorname{Int} P$ is called a formal hole of $P$, and the unbounded component of $E \backslash$ Int $P$ is called the formal exterior of $P$ and is denoted by Ext $P$.

From the above definition it follows that for any polygonal domain $P$ the sets $\operatorname{Bd} P$, Ext $P$, and any formal hole of $P$ are closed sets, while the formal interior Int $P$ is an open set.

Remark 1. We observe that the boundary bd $P$ and the interior int $P$ of the polygonal domain $P$ do not depend on the ornament Or $P$. In order to stress this situation, we call the sets bd $P$ and int $P$ the topological boundary and topological interior of $P$, respectively.

Example. For the polygonal domain $P$ shown in Fig. 1, the topological boundary bd $P$ is the union of two contours $[1,2,10,3,4,13,20,19,14,18,5,6,1]$ and $[12,17,16$,

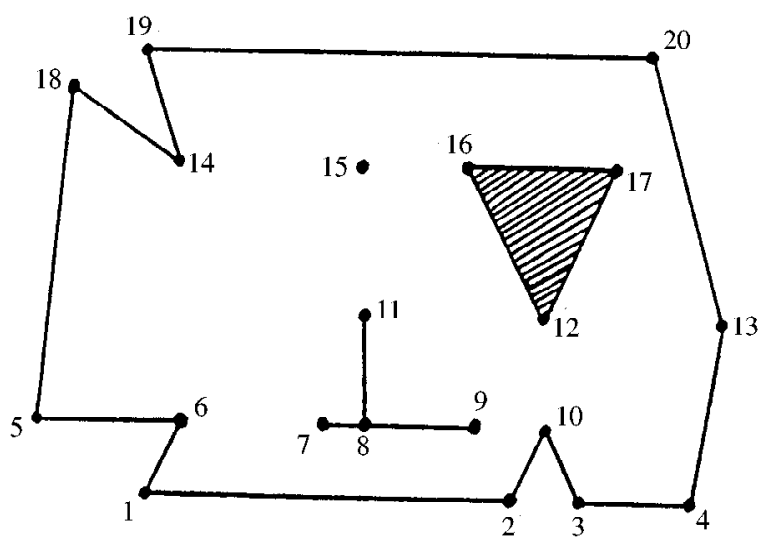

Fig. 1. A polygonal domain with degenerate holes. 
12]; Or $P$ is the union of the point $\{15\}$ and the two line segments [7,9], [8,11]. There is one topological hole, and there are three formal holes.

\section{Measure of Local Nonconvexity}

A point $x \in E$ is called a vertex of a polygonal domain $P$ if one of the following three cases holds: (i) $x$ is a vertex of a closed polygonal contour from $\mathrm{Bd} P$; (ii) $x$ is an isolated point from Or $P$; (iii) $x$ is an endpoint of one of the segments $s_{1}, s_{2}, \ldots, s_{t}$ forming Or $P$. The set of vertices of $P$ is denoted by Vert $P$. A nontrivial line segment $[x, z] \subset$ bd $P$ is called an elementary segment of $P$ provided Vert $P \cap[x, z]=\{x, z\}$.

Any vertex $v$ of a polygonal domain $P$ (unless it is an isolated point in $\mathrm{Bd} P$ ) is the apex of at least one interior angle $\angle u v w$, where $[u, v]$ and $[v, w]$ denote elementary segments of $P$. We allow the vectors $\overrightarrow{v u}$ and $\overrightarrow{v w}$ to be collinear (in this case $\angle u v w$ equals $\pi$ ). An interior angle $\angle u v w$ is called convex if it is at most $\pi$, and it is concave provided it is larger than $\pi$.

Definition 2. A vertex $v$ of a polygonal domain $P$ is called a vertex of local nonconvexity of $P$ if it is either an isolated point in $\mathrm{Bd} P$ or the apex of a concave interior angle of $P$; otherwise $v$ is called a vertex of local convexity of $P$.

Remark 2. The notion vertex of local nonconvexity of a polygonal domain is a particular case of the notion point of weak local nonconvexity of an arbitrary set (see, e.g., [16]). Recall that a point $v$ in the plane is a point of (weak) local nonconvexity of a planar set $X$ if for any neighborhood $U$ of $v$ at least one of the components of the intersection $X \cap U$ is not convex. In this context, the only points of local nonconvexity of a polygonal domain $P$ are its vertices of local nonconvexity.

The following two simple lemmas are used later.

Lemma 1. Any formal hole of a polygonal domain P contains at least one vertex of local nonconvexity of $P$.

Proof. Let $H$ be a formal hole of $P$. Then any vertex of the convex hull conv $H$ of $H$ is a point of local nonconvexity of $P$.

Lemma 2. A polygonal domain $P$ has no vertices of local nonconvexity if and only if each component of Int $P$ is an open convex polygon.

Proof. A polygonal domain $P$ has no points of local nonconvexity if and only if $\mathrm{Bd} P$ contains no isolated points and every interior angle of $P$ is convex. In this case each component of Int $P$ is an open simple polygon whose interior angles are convex, i.e., it is convex itself.

With respect to a given family $\mathcal{F}$ of directions in the plane, by a simple $\mathcal{F}$-chord of a polygonal domain $P$ we mean a closed line segment $[x, z]$ parallel to a direction from $\mathcal{F}$ such that the vertices $x, z$ belong to $\mathrm{Bd} P$ and the open line interval ] $x, z$ [ lies in Int $P$. 
Definition 3. Let $P$ be a polygonal domain. The measure $m(v)\left(=m_{\mathcal{F}}(v)\right)$ of local nonconvexity of $P$ at a vertex $v$ with respect to a given family $\mathcal{F}$ of directions is defined as follows:

(1) If $v$ is an isolated point in $\mathrm{Bd} P$, then $m(v)=2$.

(2) If $v$ is the apex of a concave interior angle $\angle u v w$ of $P$, then $m(v)$ is the minimum number of simple $\mathcal{F}$-chords of the form $[v, w]$ which divide $\angle u v w$ into convex angles.

Remark 3. >From the definitions above and from Remark 2 it follows that a point $v$ from a polygonal domain $P$ is a vertex of local nonconvexity if and only if $m(v)>0$.

Lemma 3. For any vertex $v$ of local nonconvexity of a polygonal domain $P$, one has $1 \leq m(v) \leq 2$.

Proof. Let $l$ be any direction in $\mathcal{F}$ and let $v$ be a vertex of local nonconvexity of $P$. If $v$ is an isolated point in $\operatorname{Bd} P$, then, by definition, $m(v)=2$. Assume $v$ is the apex of a concave interior angle $\angle u v w$ of $P$. Since $\angle u v w>\pi$, a simple chord $[v, z]$ of $P$, having direction $l$ and lying inside the interior of $\angle u v w$, can be drawn. If both $\angle u v z$ and $\angle z v w$ are convex, then we have $m(v)=1$. If one of these angles, say $\angle u v z$, is concave, then there is one more simple chord $[v, x]$ of $P$ in direction $l$, lying inside the interior part of $\angle u v z$. Now $\angle u v x, \angle x v z$, and $\angle z v w$ are all convex, and hence $m(v)=2$.

Definition 4. For a polygonal domain $P$, the sum

$$
m(P):=\sum m(v)
$$

taken over all vertices of local nonconvexity of $P$, is called the measure of local nonconvexity of $P$ (with respect to a given family $\mathcal{F}$ of directions).

Remark 4. If $P$ is a usual polygon with holes and $\mathcal{F}$ is the family of all directions in the plane, then it easily follows that the measure of local nonconvexity of $P$ equals one at any of its vertices of local nonconvexity. In this case $m(P)$ is exactly the number of vertices of local nonconvexity of $P$.

\section{Convex Partitions}

As usual, by a convex partition of a polygon $P$ we mean any of its decompositions into finitely many closed, nonoverlapping convex pieces. For the case of polygonal domains with degenerate holes, we obtain:

Definition 5. A polygonal domain $P$ is said to be partitioned into convex polygons $Q_{1}, Q_{2}, \ldots, Q_{r}$ if

$$
\bigcup_{i=1}^{r} \text { int } Q_{i} \subset \operatorname{Int} P \subset \bigcup_{i=1}^{r} Q_{i}, \quad \text { int } Q_{i} \cap \text { int } Q_{j}=\emptyset, \quad i \neq j .
$$


In other words, nonoverlapping convex polygons $Q_{1}, Q_{2}, \ldots, Q_{r}$ partition $P$ if their union coincides with $P$ and the formal boundary $\mathrm{Bd} P$ of $P$ lies in the union of the topological boundaries bd $Q_{1}$, bd $Q_{2}, \ldots$, bd $Q_{r}$.

The next lemma gives a method of identifying convex partitions of a polygonal domain $P$ by the process of adding some closed line segments to its formal boundary $\operatorname{Bd} P$.

Lemma 4. Let $\mathcal{Q}=\left\{Q_{1}, Q_{2}, \ldots, Q_{r}\right\}$ be a partition of a polygonal domain $P$ into simple polygons. Denote by $P_{Q}$ a new polygonal domain whose formal boundary is the union of the topological boundaries of the polygons $Q_{1}, Q_{2}, \ldots, Q_{r}$ :

$$
\operatorname{Bd} P_{Q}:=\bigcup_{i=1}^{r} \text { bd } Q_{i}, \quad \text { Int } P_{Q}:=\operatorname{int} P \backslash \operatorname{Bd} P_{Q} .
$$

Then all the polygons $Q_{1}, Q_{2}, \ldots, Q_{r}$ are convex if and only if $P_{Q}$ has no vertices of local nonconvexity.

Proof. A simple polygon $Q_{i}$ is convex if and only if its interior int $Q_{i}$ is a convex set. From the definition of $P_{Q}$ it follows that Int $P_{Q}$ is the disjoint union of the sets int $Q_{i}$, $i=1,2, \ldots, r$. Now the assertion of the lemma follows from Lemma 2 .

As we have seen above, any convex partition of a polygonal domain $P$ can be considered as the repeated addition of finitely many closed line segments to $\mathrm{Bd} P$. In the literature, such an addition of segments, which are simple chords of a polygon, is known as guillotine cutting. We give here a definition of guillotine cutting for the case of polygonal domains.

Definition 6. Let $P$ be a polygonal domain. The polygonal domain $P^{\prime}$ is said to be obtained from $P$ by guillotine cutting along a simple chord $[x, z]$ of $P$ if

$$
\operatorname{Bd} P^{\prime}:=\operatorname{Bd} P \cup[x, z] \text { and } \operatorname{Int} P^{\prime}:=\operatorname{Int} P \backslash[x, z] .
$$

Definition 7. A polygonal domain $P^{\prime}$ is said to be obtained from a polygonal domain $P$ by repeated guillotine cutting if there is a sequence $P_{0}=P, P_{1}, \ldots, P_{t}=P^{\prime}$ of polygonal domains such that $P_{i}$ is obtained from $P_{i-1}$ by guillotine cutting, $i=$ $1,2, \ldots, t$. If all cutting segments are parallel to directions from a given family $\mathcal{F}$, we speak about a repeated guillotine $\mathcal{F}$-cutting. In terms of partitions, we speak about repeated guillotine $\mathcal{F}$-cuttings as guillotine $\mathcal{F}$-partitions.

\section{Naive Convex Guillotine $\mathcal{F}$-Partition}

Here we present a convex guillotine $\mathcal{F}$-partition of a polygonal domain by means of consecutive cuttings, eliminating local nonconvexity of the domain at all points of local nonconvexity. In what follows this partition is used as a procedure of the guillotine $\mathcal{F}$-partition of a polygonal domain into a minimum number of convex pieces. 
Let $v$ be a vertex of local nonconvexity of a polygonal domain $P$, and let $[v, z]$ be a simple chord of $P$. We say that the guillotine cut of $P$ along $[x, z]$ decreases the measure $m(v)$ of local nonconvexity by one provided $m^{\prime}(v)=m(v)-1$, where $m^{\prime}(v)$ is the measure of local nonconvexity of the domain $P^{\prime}$ obtained at $v$.

Lemma 5. Let $\mathcal{F}$ be a nonempty family of directions in the plane, and let $P$ be a polygonal domain. For any vertex $v$ of local nonconvexity of $P$ there is a guillotine $\mathcal{F}$-cut decreasing $m(v)$ by one.

Proof. The proof of this lemma is similar to that of Lemma 3. If $v$ is an isolated point in $\mathrm{Bd} P$, we choose a simple $\mathcal{F}$-chord $[v, z]$ of $P$. In this case $m^{\prime}(v)=m(v)-1=1$, where $P^{\prime}$ is the polygonal domain obtained from $P$ by the guillotine cut along $[v, z]$.

If $v$ is the apex of a concave interior angle of $P$, then we choose a simple chord $[v, z]$ of $P$ parallel to a direction from $\mathcal{F}$ and lying in the interior part of this angle. It is easy to see that in this case we also have $m^{\prime}(v)=m(v)-1$.

Theorem 1. Let $\mathcal{F}$ be a nonempty family of directions in the plane, and let $P$ be a polygonal domain. Then, by repeated guillotine $\mathcal{F}$-cuts, $P$ can be partitioned into at most

$$
m+c-h
$$

convex polygons, where

$m$ is the measure of local nonconvexity of $P$,

$c$ is the number of connected components of Int $P$, and

$h$ is the number of formal holes of $P$.

Proof. First we consider the case $m=0$. Then, by Lemma 1, we also have $h=0$. Moreover, every component of Int $P$ is an open convex polygon (see Lemma 2). The closures of these polygons partition $P$ into $c$ convex pieces (in the sense of Definition 3 ). Hence in the case $m=0$ the polygonal domain $P$ is $\mathcal{F}$-partitioned by its ornament Or $P$ into $c(=m+c-h)$ convex pieces.

Now assume that $m>0$, and let $v$ be a point of local nonconvexity of $P$. By Lemma 6, there is a guillotine $\mathcal{F}$-cut of $P$ along a simple chord $[v, z]$, decreasing the measure of local nonconvexity of $P$ at $v$ by one. Put $P_{0}=P$ and denote by $P_{1}$ the polygon obtained from $P$ by this cutting. It is easy to see that either we have $h\left(P_{1}\right)=h\left(P_{0}\right)-1$ or $c\left(P_{1}\right)=c\left(P_{0}\right)+1$, i.e.,

$$
c\left(P_{1}\right)-h\left(P_{1}\right)=c\left(P_{0}\right)-h\left(P_{0}\right)+1=c-h+1 .
$$

Since the second vertex $z$ of the chord $[v, z]$ can be a point of local nonconvexity of $P_{0}$ also, we have $m\left(P_{0}\right)-2 \leq m\left(P_{1}\right) \leq m\left(P_{0}\right)-1$, and hence

$$
m\left(P_{1}\right)+c\left(P_{1}\right)-h\left(P_{1}\right) \leq m+c-h .
$$

By repeating the above procedure of guillotine $\mathcal{F}$-cutting, we obtain a sequence $P_{0}=P$, $P_{1}, \ldots, P_{k}, k \leq m$, of polygonal domains such that

$$
m\left(P_{i}\right)+c\left(P_{i}\right)-h\left(P_{i}\right) \leq m\left(P_{i-1}\right)+c\left(P_{i-1}\right)-h\left(P_{i-1}\right), \quad i=1,2, \ldots, k,
$$


and $P_{k}$ has no points of local nonconvexity. As in the case $m(P)=0$ we have $h\left(P_{k}\right)=0$, and $\mathrm{Bd} P_{k}$ cuts Int $P_{k}$ into $c\left(P_{k}\right)$ open convex pieces. Since

$$
c\left(P_{k}\right)=m\left(P_{k}\right)+c\left(P_{k}\right)-h\left(P_{k}\right) \leq m+c-h,
$$

we obtain the required $\mathcal{F}$-partition.

\section{Compatible Families of Diagonals}

Let $\mathcal{F}$ be a certain family of directions in the plane, and let $P$ be a polygonal domain. For any family

$$
\mathcal{L}=\left\{l_{1}, l_{2}, \ldots, l_{r}\right\}, \quad l_{i}=\left[a_{i}, b_{i}\right], \quad i=1,2, \ldots, r,
$$

of noncrossing $\mathcal{F}$-diagonals of $P$, we denote by $P_{L}$ (resp. by $R_{i}$ ) the polygonal domain obtained from $P$ by the addition of the set $L=l_{1} \cup l_{2} \cup \cdots \cup l_{r}$ (resp. of the set $L \backslash l_{i}$ ) to Bd $P$. We also put

$$
V(\mathcal{L})=\left\{a_{1}, b_{1}, a_{2}, b_{2}, \ldots, a_{r}, b_{r}\right\}
$$

and we denote by $\operatorname{deg}_{L}(w)$ the number of diagonals in $\mathcal{L}$ incident to a vertex $w \in \mathcal{L}$.

Definition 8. A family (1) of noncrossing $\mathcal{F}$-diagonals of a polygonal domain $P$ is called a compatible family of $\mathcal{F}$-diagonals provided

$$
m_{i}\left(a_{i}\right)=m_{L}\left(a_{i}\right)+1, \quad m_{i}\left(b_{i}\right)=m_{L}\left(b_{i}\right)+1
$$

for all $i=1,2, \ldots, r$, where $m_{L}(v)$ (resp. $\left.m_{i}(v)\right)$ is the measure of local nonconvexity of the domain $P_{L}$ (resp. of the domain $\left.R_{i}\right)$ at a vertex $v\left(\in\left\{a_{i}, b_{i}\right\}\right)$.

Lemma 6. Let $r_{1}, r_{2}, \ldots, r_{k}, k \geq 5$, be some rays with a common apex a, taken in clockwise order around a (possibly, $r_{k}=r_{1}$ ). Then there is an integer $i \in\{1,2, \ldots, k-2\}$ such that the rays $r_{i}$ and $r_{i+2}$ form a convex angle.

Proof. Let $\lambda_{i}, i=1,2, \ldots, k-1$, be the oriented angle formed by $r_{i}$ and $r_{i+1}$. It is sufficient to find an integer $i \in\{1,2, \ldots, k-2\}$ such that $\lambda_{i}+\lambda_{i+1} \leq \pi$. Since

$$
\left(\lambda_{1}+\lambda_{2}\right)+\left(\lambda_{k-2}+\lambda_{k-1}\right) \leq \lambda_{1}+\lambda_{2}+\cdots+\lambda_{k-1} \leq 2 \pi,
$$

one of the sums $\lambda_{1}+\lambda_{2}, \lambda_{k-2}+\lambda_{k-1}$ is at most $\pi$.

Lemma 7. Let (1) be a compatible family of diagonals of a polygonal domain $P$, and let $P_{L}$ be the polygonal domain obtained from $P$ by adding the set $l_{1} \cup l_{2} \cup \cdots \cup l_{r}$ to Bd $P$. Then:

(1) The set $V(\mathcal{L})$ consists of vertices of local nonconvexity of $P$.

(2) For any vertex $w \in V(\mathcal{L})$ one has $m_{L}(w)<m(w)$ and $1 \leq \operatorname{deg}_{L}(w) \leq 3$. 
(3) If a vertex $w \in V(\mathcal{L})$ is not an isolated point in $\mathrm{Bd} P$, then $\operatorname{deg}_{L}(w) \leq 2$.

(4) If one has $m_{L}(w)=1$ for a vertex $w \in V(\mathcal{L})$, then $\operatorname{deg}_{L}(w)=1$.

Proof. Let $w$ be any point in $V(\mathcal{L})$. According to the definitions above, we have $m(v) \geq$ $m_{i}(v)=m_{L}(v)+1$. This implies $m(w)>m_{L}(w)$ as well as the local nonconvexity of $P$ at the vertex $v$ (see Remark 3 ).

We prove the inequality $1 \leq \operatorname{deg}_{L}(w) \leq 3$. Since $w$ is an apex of at least one segment from $\mathcal{L}$, the case $1 \leq \operatorname{deg}_{L}(w)$ is trivial. To prove the inequality $\operatorname{deg}_{L}(w) \leq 3$, we distinguish two cases depending on whether or not $w$ is an isolated point in $\mathrm{Bd} P$. Since $m(w) \leq 2$ (see Lemma 3 ), the above statements imply that $0 \leq m_{L}(w) \leq 1$.

(i) First we assume that $w$ is an isolated point in $\mathrm{Bd} P$.

If $m_{L}(w)=0$, then the $\mathcal{F}$-diagonals from $\mathcal{L}$ incident to $w$ divide the complete angle with apex $w$ (i.e., the angle of $2 \pi$ ) into convex angles. If we delete from $\mathcal{L}$ any of these diagonals, say $l_{i}$, then from (2) it follows that $w$ becomes a vertex of local nonconvexity in $P_{i}$, i.e., a concave inner angle with apex $w$ appears. Based on Lemma 6 we conclude that the number of such diagonals is at most three, i.e., $\operatorname{deg}_{L}(w) \leq 3$.

If $m_{L}(w)=1$, then $w$ is the apex of a concave inner angle $\alpha$ of the domain $P_{L}$. We claim that $\alpha$ has $2 \pi$ and is formed by a single $\mathcal{F}$-diagonal from $\mathcal{L}$. Indeed, assume that $\alpha=\angle b_{p} w b_{q}$, where $l_{p}=\left[w, b_{p}\right]$ and $l_{q}=\left[w, b_{q}\right]$ are two distinct $\mathcal{F}$-diagonals (with $\left.a_{p}=a_{q}=w\right)$. If we delete $l_{p}$ from $\mathrm{Bd} P_{L}$, then, as is easily seen, we obtain $m_{p}(w)=1$, contradicting condition (2) for $i=p$. Hence $w$ is incident to exactly one $\mathcal{F}$-diagonal from $\mathcal{L}$, i.e., $\operatorname{deg}_{L}(w)=1$.

(ii) Now we assume that $w$ is the apex of a concave inner angle $\angle u w v$ of $P$, formed by the elementary segments $[u, w],[v, w] \subset \operatorname{Bd} P$.

If $m_{L}(w)=0$, then the $\mathcal{F}$-diagonals from $\mathcal{L}$ incident to $w$ divide $\angle u w v$ into convex angles. If we delete from $\mathcal{L}$ any of these diagonals, say $l_{i}$, then from (2) it follows that $w$ becomes a vertex of local nonconvexity in $P_{i}$, i.e., a concave inner angle of $P_{i}$ with apex $w$ appears. From Lemma 6 we conclude that $\operatorname{deg}_{L}(w) \leq 2$.

If $m_{L}(w)=1$, then $w$ is the apex of a concave inner angle $\alpha$ of the domain $P_{L}$. We claim that $\alpha$ is formed by a simple chord $[u, w] \subset \operatorname{Bd} P$ and an $\mathcal{F}$-diagonal from $\mathcal{L}$.

Indeed, if $\alpha$ is formed by two simple chords of $P$, then all $\mathcal{F}$-diagonals from $\mathcal{L}$ incident to $w$ are contained in the convex angle complementary to $\alpha$. In this case we can delete from $\mathcal{L}$ any of these diagonals, say $l_{i}$. Since $m_{i}(w)=1$, we obtain a contradiction with (2).

If $\alpha$ is formed by two $\mathcal{F}$-diagonals, say $l_{p}=\left[w, b_{p}\right]$ and $l_{q}=\left[w, b_{q}\right]$ from $\mathcal{L}$, we can delete $l_{p}$ from $\mathrm{Bd} P_{L}$, again obtaining $m_{p}(w)=1$, which is impossible by (2).

Hence $\alpha$ is formed by a simple chord $[u, w] \subset \operatorname{Bd} P$ and an $\mathcal{F}$-diagonal from $\mathcal{L}$. By the same argument, no other $\mathcal{F}$-diagonal incident to $w$ can intersect the interior of the angle complementary to $\alpha$. Thus $\operatorname{deg}_{L}(w)=1$.

It remains to observe that items (3) and (4) of the assertion in Lemma 7 are proved within cases (i) and (ii) above.

Definition 9. The effective number $e(\mathcal{L})$ of a compatible family (1) of diagonals of a polygonal domain $P$ is defined by

$$
e(\mathcal{L})=r+\sum \min \left\{m(w)-\operatorname{deg}_{L}(w), 0\right\}
$$


where the sum is taken over all vertices $w \in V(\mathcal{L})$, and the effective number $e(P)$ of $P$ is defined by

$$
e(P)=\max e(\mathcal{L}),
$$

where the maximum is taken over all compatible families $\mathcal{L}$ of diagonals of $P$.

Example. If a family $\mathcal{F}$ consists of two directions parallel to the coordinate axes of the plane, then the polygonal domain $P$ in Fig. 1 (with one two-dimensional and two degenerate holes) has the effective number $e(P)=4$, attained at the compatible family $\mathcal{L}=\{[1,2],[3,4],[5,6],[6,7]\}$.

Remark 5. If $P$ is a usual polygon with holes and $\mathcal{F}$ is the family of all directions in the plane, then for any compatible family $\mathcal{L}$ of diagonals of $P$ its effective number $e(\mathcal{L})$ equals card $V(\mathcal{L})-\operatorname{card} \mathcal{L}$ (see Remark 4$)$.

Lemma 8. Let (1) describe a compatible family $\mathcal{L}$ of diagonals of a polygonal domain $P$, and let $P_{L}$ be the polygonal domain obtained from $P$ by adding the set $l_{1} \cup l_{2} \cup \cdots \cup l_{r}$ to $\mathrm{Bd} P$. Then

$$
m(P)-m\left(P_{L}\right)=r+e(\mathcal{L}) .
$$

Proof. First we show that, for any vertex $w \in V(\mathcal{L})$, we have

$$
m(w)-m_{L}(w)=\operatorname{deg}_{L}(w)+\min \left\{m(w)-\operatorname{deg}_{L}(w), 0\right\} .
$$

To do this, we distinguish some different cases.

Case 1: $m(w)=1$ and $m_{L}(w)=0$. Then

$$
\begin{aligned}
m(w)-m_{L}(w) & =1=\operatorname{deg}_{L}(w)+\left(m(w)-\operatorname{deg}_{L}(w)\right) \\
& =\operatorname{deg}_{L}(w)+\min \left\{m(w)-\operatorname{deg}_{L}(w), 0\right\} .
\end{aligned}
$$

Case 2: $m(w)=2$ and $m_{L}(w)=1$. Then $\operatorname{deg}_{L}(w)=1($ see Lemma 7) and

$$
m(w)-m_{L}(w)=1=\operatorname{deg}_{L}(w)+\min \left\{m(w)-\operatorname{deg}_{L}(w), 0\right\} .
$$

Case 3: $m(w)=2$ and $m_{L}(w)=0$. Then $\operatorname{deg}_{L}(w) \geq 2$ and

$$
\begin{aligned}
m(w)-m_{L}(w) & =2=\operatorname{deg}_{L}(w)+\left(m(w)-\operatorname{deg}_{L}(w)\right) \\
& =\operatorname{deg}_{L}(w)+\min \left\{m(w)-\operatorname{deg}_{L}(w), 0\right\} .
\end{aligned}
$$

Now

$$
\begin{aligned}
m(P)-m\left(P_{L}\right) & =\sum\left\{m(w)-m_{L}(w): w \in \operatorname{Vert} P\right\} \\
& =\sum\left\{m(w)-m_{L}(w): w \in V(\mathcal{L})\right\} \\
& =\sum\left\{\operatorname{deg}_{L}(w)+\min \left\{m(w)-\operatorname{deg}_{L}(w), 0\right\}: w \in V(\mathcal{L})\right\} \\
& =2 r+\sum\left\{\min \left\{m(w)-\operatorname{deg}_{L}(w), 0\right\}: w \in V(\mathcal{L})\right\} \\
& =r+e(\mathcal{L})
\end{aligned}
$$




\section{Minimum Convex Guillotine $\mathcal{F}$-Partition}

This section is devoted to the proof of the theorem describing the main result of this paper.

Theorem 2. Let $\mathcal{F}$ be a certain family of directions in the plane, and let $P$ be a polygonal domain. The minimum number of pieces in a convex guillotine $\mathcal{F}$-partition of $P$ equals

$$
m+c-h-e,
$$

where

$m$ is the measure of local nonconvexity of $P$,

$c$ is the number of connected components of Int $P$,

$h$ is the number of formal holes of $P$, and

$e$ is the effective number of $P$.

Example. Figure 2 shows a minimum convex partition of a polygonal domain $P$, where guillotine cuts along the directions of the coordinate axes in the plane are used. Here $m(P)=12, c(P)=1, h(P)=3, e(P)=4$, and the minimum number of convex pieces equals $6(=12+1-3-4)$.

Proof of Theorem 2. Let $\mathcal{L}=\left\{l_{1}, l_{2}, \ldots, l_{r}\right\}$ be an effective family of diagonals of $P$ with the effective number $e(\mathcal{L})=e(P)$. As above, we denote by $P_{L}$ the polygonal domain obtained from $P$ by adding the set $l_{1} \cup l_{2} \cup \cdots \cup l_{r}$ to $\mathrm{Bd} P$. Since this addition can be considered as the repeated guillotine cutting along $l_{1}, l_{2}, \ldots, l_{r}$, and since any cut $l_{i}$ either increases the number of components of the formal interior by one or decreases the number of formal holes by one, we have

$$
c\left(P_{L}\right)-h\left(P_{L}\right)=c-h+r .
$$

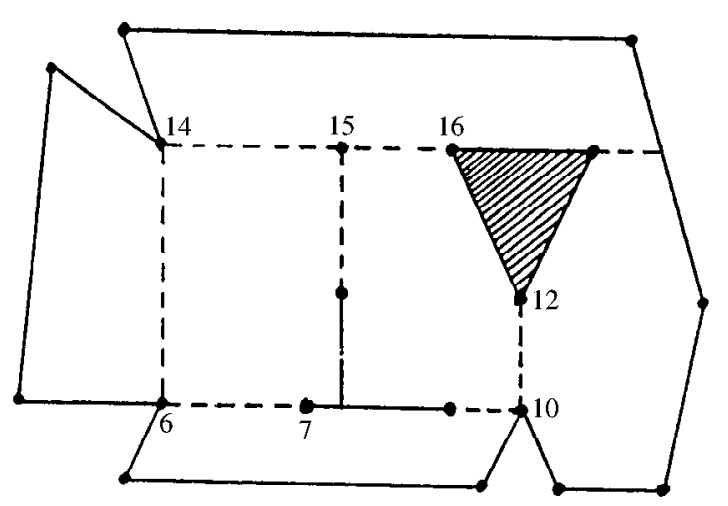

Fig. 2. Minimum convex guillotine partition along two directions. 
Due to Lemma 8,

$$
m\left(P_{L}\right)+c\left(P_{L}\right)-h\left(P_{L}\right)=m+c-h-e(\mathcal{L})=m+c-h-e .
$$

Now, applying the naive guillotine $\mathcal{F}$-cutting procedure described in Theorem 1 to the domain $P_{L}$, we obtain a convex guillotine $\mathcal{F}$-partition of $P$ into at most $m+c-h-e$ convex polygons.

It remains to prove that any convex guillotine $\mathcal{F}$-partition contains at least $m+c-h-e$ pieces. Let $P_{0}=P, P_{1}, \ldots, P_{t}=P^{\prime}$ be a sequence of polygonal domains such that (1) $P_{i}$ is obtained from $P_{i-1}$ by a guillotine $\mathcal{F}$-cutting along a simple chord $l_{i}=\left[a_{i}, b_{i}\right]$, $i=1,2, \ldots, t$, and (2) the formal interior Int $P^{\prime}$ of the last domain $P^{\prime}$ is dissected by its formal boundary $\mathrm{Bd} P^{\prime}$ into, say $s$, convex open polygons (see Lemma 4). In particular, we have $m\left(P^{\prime}\right)=0, c\left(P^{\prime}\right)=s$, and $h\left(P^{\prime}\right)=0$.

We prove the inequality $s \geq m+c-h-e$. For this purpose, in the family $\mathcal{M}=$ $\left\{l_{1}, l_{2}, \ldots, l_{t}\right\}$ a compatible subfamily of diagonals of $P$ are selected. Before doing this, we make one auxiliary observation. As before, we denote by $R_{i}$ the polygonal domain obtained from $P$ by the addition of the set $l_{1} \cup l_{2} \cup \cdots \cup l_{i-1} \cup l_{i+1} \cup \cdots \cup l_{t}$ to $\mathrm{Bd} P$, and by $m^{\prime}(w)$ (resp. by $m_{i}(w)$ ) the measure of local nonconvexity of the polygon $P^{\prime}$ (resp. of $R_{i}$ ) at a vertex $w \in \operatorname{Vert} P$.

Observation. If for an $\mathcal{F}$-chord $l_{i}=\left[a_{i}, b_{i}\right]$ both equalities

$$
m_{i}\left(a_{i}\right)=m^{\prime}\left(a_{i}\right)+1, \quad m_{i}\left(b_{i}\right)=m^{\prime}\left(b_{i}\right)+1
$$

hold, then $l_{i}$ is a diagonal of $P$. Indeed, if $a_{i}$, say, is not a vertex of $P$, then it is a point of local convexity of $P$ (see Remark 2). In this case, as is easy to see, $a_{i}$ is a point of local convexity of any polygonal domain $R_{i}$, contradicting (3).

To select a compatible subfamily of diagonals of $P$ in $\mathcal{M}$, we repeatedly use the following procedure.

Deletion of Noneffective Diagonals. We consider the sequence $l_{t}, l_{t-1}, \ldots, l_{1}$ (in this order) and, starting from $l_{t}$, verify for each $l_{i}$ whether or not both the equalities in (3) hold. If all the $2 t$ equalities in (3) hold then, by the Observation above, we see that $\mathcal{M}$ is a compatible family of $\mathcal{F}$-diagonals of $P$. Assume that not all of these $2 t$ equations hold, and let $i$ be the largest integer in $\{1,2, \ldots, t\}$ which violates at least one of the equalities in (3). Since $m_{i}(w) \geq m^{\prime}(w)$ for any vertex $w \in \operatorname{Vert} P$, at least one of the equalities

$$
m_{i}\left(a_{i}\right)=m^{\prime}\left(a_{i}\right), \quad m_{i}\left(b_{i}\right)=m^{\prime}\left(b_{i}\right)
$$

holds. As was simultaneously mentioned above, we have either $c\left(R_{i}\right)=c\left(P^{\prime}\right)-1$ or $h\left(R_{i}\right)=h\left(P^{\prime}\right)+1$. Hence

$$
\begin{aligned}
s & =m\left(P^{\prime}\right)+c\left(P^{\prime}\right)-h\left(P^{\prime}\right) \geq\left(m\left(R_{i}\right)-1\right)+\left(c\left(R_{i}\right)-h\left(R_{i}\right)+1\right) \\
& =m\left(R_{i}\right)+c\left(R_{i}\right)-h\left(R_{i}\right) .
\end{aligned}
$$

By the Observation above, all the chords $l_{t}, l_{t-1}, \ldots, l_{i+1}$ are diagonals of $P$. Hence $R_{i}$ can be considered as the polygonal domain obtained from $P$ by a repeated $\mathcal{F}$-cutting 
along simple chords $l_{1}, l_{2}, \ldots, l_{i-1}, l_{i+1}, \ldots, l_{t}$. We delete $l_{i}$ from the family $\mathcal{M}$ and renumber the sequence $l_{1}, l_{2}, \ldots, l_{i-1}, l_{i+1}, \ldots, l_{t}$ by the indices $1,2, \ldots, t-1$, respectively. Furthermore, we put $\mathcal{M}=\left\{l_{1}, l_{2}, \ldots, l_{t-1}\right\}$ and $P^{\prime}=P_{t-1}$. Since $P^{\prime}=R_{i}$, we have $m\left(P^{\prime}\right)+c\left(P^{\prime}\right)-h\left(P^{\prime}\right) \leq s$.

After repeated application of the above procedure we obtain a subfamily $\mathcal{N}=$ $\left\{l_{1}, l_{2}, \ldots, l_{q}\right\} \subset \mathcal{L}$ of $\mathcal{F}$-chords of $P$ such that no chord $l_{i} \in \mathcal{N}$ violates any of the equalities in (3). This means that $\mathcal{N}$ is a compatible family of diagonals of $P$. Denote by $P_{N}$ the polygonal domain obtained from $P$ by the set $l_{1} \cup l_{2} \cup \cdots \cup l_{q}$ to $\operatorname{Bd} P$. By the argument in the procedure above,

$$
m\left(P_{N}\right)+c\left(P_{N}\right)-h\left(P_{N}\right) \leq s .
$$

As was shown at the beginning of the proof of Theorem 2, we have

$$
m+c-h-e \leq m+c-h-e(\mathcal{N})=m\left(P_{N}\right)+c\left(P_{N}\right)-h\left(P_{N}\right) \leq s .
$$

\section{Related Results and Applications}

The authors do not know of any references which refer explicitly to the minimum number of convex guillotine partitions. However, besides the papers mentioned in the introduction there also exist some related results.

The well-known paper [2] contains a particular case of our Theorem 1, namely, for the case when $\mathcal{F}$ is the family of all directions in the plane and $P$ is a simple polygon (i.e., having no holes). Formally, [2], [15], and [17] deal with minimum numbers of arbitrary convex partitions (with $\mathcal{F}$ being the family of all directions in [2], and with $\mathcal{F}$ as the family of directions determined by the sides of a polygon $P$ in [15] and [17]). The formulas contained in these three papers refer to compatible families of tree-like unions of line segments, and it is not clear how to deduce the formula contained in Theorem 2 above from the results presented in [2], [15], and [17]. More precisely, the problem of minimum convex guillotine cutting for polygonal domains is (to the best of our knowledge) a new one and cannot be deduced from known results.

On the other hand, relations to [18] are very interesting. Formally, this paper deals with the minimum numbers of arbitrary convex partitions of rectilinear polygons in the directions of the coordinate axes. It is proved in [18] that among all such minimum partitions there is a guillotine one. Bearing this in mind, the main result of [18] can be deduced from our Theorem 2, which is of a more general nature.

For computational aspects, we refer to the very recent contribution [13]. For example, it is proved there that the minimal convex partition of polygons by guillotine cuts with respect to a direction family $\mathcal{F}$ is NP-hard if $|\mathcal{F}| \geq 3$, and is polynomially solvable in time $O\left(n^{3 / 2} \log n\right)$ if $|\mathcal{F}| \leq 2$. The question of the computational complexity of such partitions for the case $|\mathcal{F}| \geq 3$ and where $P$ is a simple polygon is open and seems to be interesting.

Finally, we give a few remarks on the practical applications of guillotine partitions. Optimal cutting procedures have various applications, such as pallet loading and cutting stock problems (see [5]). Depending on practical demands (such as restrictions on the 
shape of objects, position of the cuts, etc.), in many cases branch-and-bound methods or recurrent formulas of dynamic programming are used to compute patterns which are optimal in this context. It turns out that here especially the use of guillotine cuts plays an important role. There are two main reasons. Namely, sometimes guillotine cuts yield the only or the most convenient approach to optimal patterns (see, e.g., [7]-[9]) and, on the other hand, in several cases they reflect the real practical situation in the wood industry, steel industry, etc. (see [20]).

\section{References}

1. Asano, T., Asano, T., Minimum partition of polygonal region into trapezoids, 24th Ann. Symp. on Foundations of Computer Science, Silver Spring, MD, 1983, pp. 233-241.

2. Chazelle, B., Dobkin, D.P., Optimal convex decomposition, in: Computational Geometry (G.T. Toussaint, Ed.), pp. 63-133, North-Holland, Amsterdam, 1985.

3. Chazelle, B., Palios, L., Decomposition algorithms in geometry, in: Algebraic Geometry and Applications (C.L. Bajaj, Ed.), pp. 419-449, Springer-Verlag, New York, 1994.

4. Chiuyang, C., Ruei-Chuan, C., On the minimality of polygon triangulation, BIT 30 (1990), 570-582.

5. Dyckhoff, H., Finke U., Cutting and Packing in Production and Distribution. Physica-Verlag, Heidelberg, 1992.

6. Ferrari, L., Sancar, P.V., Sklansky, J., Minimum rectilinear partitions of digitized blobs, Comput. Vision Graphics Image Process. 28 (1984), 58-71.

7. Gilmore, P.C., Gomory, R.E., A linear programming approach to the cutting stock problem, Oper. Res. 9 (1961), 849-859.

8. Gilmore, P.C., Gomory, R.E., A linear programming approach to the cutting stock problem, II, Oper. Res. 11 (1963), 863-887.

9. Gilmore, P.C., Gomory, R.E., Multistage cutting stock problems of two and more dimensions, Oper. Res. 13 (1965), 94-120.

10. Keil, J.M., Sack, J.-R., Minimum decomposition of polygonal objects, in: Computational Geometry (G.T. Toussaint, Ed.), pp. 197-216, North-Holland, Amsterdam, 1985.

11. Korneenko, N.M., Matveev, G.V., Metel'skiı̌, N.N., Tyškevič, R.I., Partitions of polygons (Russian), Vesci Akad. Navuk BSSR. Ser. Fiz.-Mat. Navuk, 1978, No. 2, 25-29.

12. Lee, D.T., Preparata, F.P., Computational geometry-a survey, IEEE Trans. Comput. 33 (1984), 1072-1101.

13. Lingas, A., Soltan, V., Minimum convex partition of a polygon with holes by cuts in given directions, Manuscript, 33 pp. (1996), submitted.

14. O'Rourke, J., Computational Geometry in C, Cambridge University Press, Cambridge, 1994.

15. Prisăcaru, Ch., Soltan, P., Partition of a planar domain into $d$-convex parts and its application (Russian), Dokl. Akad. Nauk SSSR 262 (1982), 271-273. (English transl.: Soviet Math. Dokl. 25 (1982), 53-55.)

16. Saskatered, R., Straus, E.G., Valentine, F.A., A generalization of a theorem of Tietze and local convexity, J. London Math. Soc. 36 (1961), 52-56.

17. Soltan, V., Partition of a planar set into a finite number of $d$-convex parts (Russian), Kibernetika (Kiev), 1984, No. 6, 70-74. (English transl.: Cybernetics 20 (1984), 855-860.)

18. Soltan, V., Gorpinevich, A., Minimum dissection of rectilinear polygon with arbitrary holes into rectangles, Discrete Comput. Geom. 9 (1993), 57-79.

19. Soltan, V., Gorpinevich, A., Minimum number of trapezoids partitioning a polygonal region, Ann. Univ. Sci. Budapest Eötvös Sect. Math. 36 (1993), 47-60.

20. Terno, J., Lindemann, R., Scheithauer, G., Zuschnittprobleme und ihre praktische Lösung, Verlag Harry Deutsch, Thun und Frankfurt a. M., 1987.

Received January 5, 1996, and in revised form December 4, 1996. 